\title{
THE PERIODIC ARRAY OF INTERFACE CRACKS AND THEIR INTERACTION
}

\author{
David Schmueser and Maria Comninou \\ Department of Applied Mechanics and Engineering Science, University of Michigan, Ann Arbor, MI 48109, \\ U.S.A.
}

(Received 2 November 1978; in revised form February 1979)

\begin{abstract}
Abtract-A periodic array of interface cracks is considered as a simple model for studying interface crack interaction. The novel feature of the solution is the elimination of the oscillatory singularities and of the material overtapping which is accomplished by allowing frictionless contact zones at the crack tips. The results are obtained for a combination of shear and tension-compression loads applied at infinity. It is shown that the stress intnsity factors become smaller with decreasing distance between the cracks. This indicates that the spreading of an interface crack is inhibited by the presence of adjacent cracks.
\end{abstract}

\section{INTRODUCTION}

Interface flaws and cracks are of fundamental importance in the fields of composite materials, rock mechanics and hydraulic fracture. Depending on the application, the growth or coalescence of pre-existing cracks must either be prevented or enhanced and it is imperative to know whether the interacting stress fields lead to mutual attraction or repulsion of the cracks. A comparison of the stress intensity factors of the interacting cracks with those of a single crack may give an indication. Thus, the natural problem to study first is the interaction of two interface cracks. For the purpose of gaining insight, however, the computationally simpler problem of an array of periodic interface cracks is considered in the present paper.

The interaction of interface cracks is by no means a new problem. One should mention the work of Erdogan $[1,2]$ who considered various crack configurations and loading conditions. Unfortunately, in his solutions the effect of material overlapping was dismissed as confined to extremely small zones near the crack tips and thus insignificant. This misconception was prevalent in the literature, with the exception of Willis [3], until recently [5]. Moreover, it was thought that any attempt to correct the situation must necessarily involve a multitude of alternating separation and contact zones crowded at the crack tips so as to simulate the oscillatory nature of the stress fields present in the wrong solution. Both notions, albeit false, discouraged work on the problem. In [4] Comminou showed that only a single zone at each tip is required. In [5] and [6] it was found that under shear or combined shear and tensile loading, the contact zone at one of the tips is quite large and affects the global nature of the solution. In the light of these developments, the interaction of interface cracks is presently reconsidered.

\section{FORMULATION}

Consider a periodic array of interface cracks with period $2 h$ lying at the interface between two elastic solids with shear moduli $\mu_{1}, \mu_{2}$ and Poisson's ratios $\nu_{1}, \nu_{2}$ as shown in Fig. 1. Under the action of uniform shear stress $S$ and tension $T$ applied at infinity, a representative crack of length $2 L$ opens over an interval $(-a, b)$. Its two faces are in frictionless contact at the tips over the intervals $(-L,-a)$ and $(b, L)$. The parameters $a$ and $b$ are unknowns of the problem. The boundary conditions require that the shear tractions vanish over the entire length of each crack and the normal tractions vanish over the open part of each crack. To enforce these conditions, we follow the analysis of $[5]$ and [6] and define the periodic dislocation distributions $B_{x}(x)$ and $B_{y}(x)$ over the entire length and the open part of each crack, respectively. The dislocation distributions are related to the gap $g(x)$ between the solids and their relative slip $h(x)$

$$
\begin{aligned}
& g(x)=u_{y}^{(2)}(x, 0)-u_{y}^{(1)}(x, 0) \\
& h(x)=u_{x}^{(2)}(x, 0)-u_{x}^{(1)}(x, 0)
\end{aligned}
$$




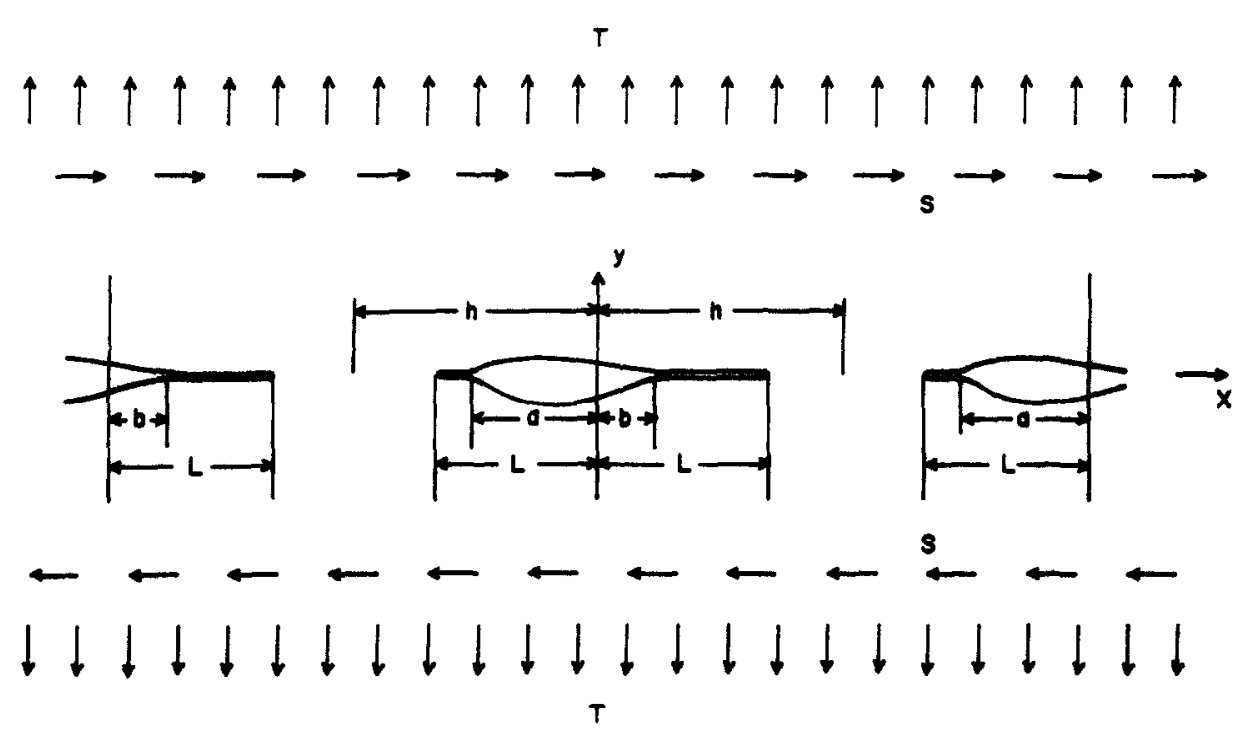

Fig. 1. The periodic array of interface cracks.

by

$$
B_{x}(x)=-\frac{\mathrm{d} h(x)}{\mathrm{d} x}, \quad B_{y}(x)=-\frac{\mathrm{d} g(x)}{\mathrm{d} x} .
$$

Following [5] or [6] we obtain the integral equations

$$
S+C\left[\beta B_{y}(x)-\frac{1}{\pi} \int_{-\infty}^{\infty} \frac{B_{x}(\xi)}{\xi-x} \mathrm{~d} \xi\right]=0
$$

over the entire length of each crack, and

$$
T-C\left[\beta B_{x}(x)+\frac{1}{\pi} \int_{-\infty}^{\infty} \frac{B_{y}(\xi)}{\xi-x} \mathrm{~d} \xi\right]=0
$$

over the open part of each crack, where

$$
C=2 \mu_{1}(1+\alpha) /\left[\left(\kappa_{1}+1\right)\left(1-\beta^{2}\right)\right], \quad \beta=\left[\mu_{2}(\kappa-1)-\mu_{1}\left(\kappa_{2}-1\right)\right] /\left[\mu_{2}\left(\kappa_{1}+1\right)+\mu_{1}\left(\kappa_{2}+1\right)\right],
$$

and $\alpha$ is defined in [4].

Using periodicity, the integrals in (4) and (5) are replaced by

$$
\begin{aligned}
\int_{-\infty}^{\infty} \frac{B_{x}(\xi)}{\xi-x} \mathrm{~d} \xi & =\int_{-L}^{L} B_{x}(\xi) \sum_{n=-\infty}^{\infty} \frac{1}{\xi-x+2 h n} \mathrm{~d} \xi \\
& =\int_{-L}^{L} B_{x}(\xi)\left[\frac{1}{\xi-x}+\sum_{n=1}^{\infty} \frac{2(\xi-x)}{(\xi-x)^{2}-(2 h n)^{2}}\right] \mathrm{d} \xi \\
\int_{-\infty}^{\infty} \frac{B_{y}(\xi)}{\xi-x} \mathrm{~d} \xi & =\int_{-a}^{b} B_{y}(\xi) \sum_{n=-\infty}^{\infty} \frac{1}{\xi-x+2 h n} \mathrm{~d} \xi \\
& =\int_{-a}^{b} B_{y}(\xi)\left[\frac{1}{\xi-x}+\sum_{n=1}^{\infty} \frac{2(\xi-x)}{(\xi-x)^{2}-(2 h n)^{2}}\right] .
\end{aligned}
$$

The series in (6) and (7) are summed by means of the identity [7]

$$
\frac{1}{x}+\sum_{n=1}^{\infty} \frac{2 x}{x^{2}-n^{2}}=\pi \cot \pi x
$$


Substituting in (4) and (5), we obtain

$$
\begin{aligned}
& S+C\left\{\beta B_{y}(x)-\frac{1}{2 h} \int_{-L}^{L} B_{x}(\xi) \cot \left[\frac{\pi(\xi-x)}{2 h}\right] \mathrm{d} \xi\right\}=0,-L<x<L \\
& T-C\left\{\beta B_{x}(x)+\frac{1}{2 h} \int_{-a}^{b} B_{y}(\xi) \cot \left[\frac{\pi(\xi-x)}{2 h}\right] \mathrm{d} \xi\right\}=0,-a<x<b .
\end{aligned}
$$

To ensure single-valued displacements, we require in addition that

$$
\int_{-L}^{L} B_{x}(\xi) \mathrm{d} \xi=0, \quad \int_{-a}^{b} B_{y}(\xi) \mathrm{d} \xi=0
$$

The integral eqns (9) and (10) can be transformed to equations with explicit Cauchy kernels by means of the following changes of variables

$$
\begin{aligned}
x & =L x^{\prime}, & & \xi=L \xi^{\prime} \\
\tan \left(\frac{\pi L \xi^{\prime}}{2 h}\right) & =u d, & & \tan \left(\frac{\pi L x^{\prime}}{2 h}\right)=s d
\end{aligned}
$$

where

$$
d=\tan \left(\frac{\pi L}{2 h}\right)
$$

Thus, (9) and (10) become

$$
\begin{array}{ll}
S+C\left\{\beta B_{y}(s)-\frac{1}{\pi} \int_{-1}^{1} \frac{B_{x}(u)\left(1+d^{2} u s\right)}{(u-s)\left(1+d^{2} u^{2}\right)} \mathrm{d} u\right\}=0, & -1<s<1 \\
T-C\left\{\beta B_{x}(s)+\frac{1}{\pi} \int_{\gamma_{1}}^{\gamma_{2}} \frac{B_{y}(u)\left(1+d^{2} u s\right)}{(u-s)\left(1+d^{2} u^{2}\right)} \mathrm{d} u\right\}=0, & \gamma_{1}<s<\gamma_{2}
\end{array}
$$

where

$$
\gamma_{1}=-\frac{1}{d} \tan \left(\frac{\pi a}{2 h}\right), \quad \gamma_{2}=\frac{1}{d} \tan \left(\frac{\pi b}{2 h}\right)
$$

and the same symbols for the functions were retained in the new variables. Introducing the new functions

$$
\psi(u)=\frac{B_{x}(u)}{1+d^{2} u^{2}}, \quad \phi(u)=\frac{B_{y}(u)}{1+d^{2} u^{2}},
$$

(11) and (12) become

$$
\int_{-1}^{1} \psi(u) \mathrm{d} u=0, \quad \int_{\gamma_{1}}^{\gamma_{2}} \phi(u) \mathrm{d} u=0
$$

Using (19)-(22), (16) and (17) may be written as

$$
\begin{aligned}
& \frac{1}{\pi} \int_{-1}^{1} \frac{\psi(u)}{u-s} \mathrm{~d} u=\beta \phi(s)+\frac{S}{C\left(1+d^{2} s^{2}\right)} \\
& \beta \psi(s)+\frac{1}{\pi} \int_{\gamma_{1}}^{\gamma_{2}} \frac{\phi(u)}{u-s} \mathrm{~d} u=\frac{T}{C\left(1+d^{2} s^{2}\right)}
\end{aligned}
$$


Equation (23) can be viewed as a Cauchy integral equation for the unknown function $\psi(u)$ treating $\phi(s)$ as known. The solution, allowing integrable singularities in $\psi(u)$ at -1 and 1 and with (21) enforced, is [8]:

$$
\psi(s)=\left(1-s^{2}\right)^{-1 / 2}\left\{\frac{S s\left(1+d^{2}\right)^{1 / 2}}{C\left(1+d^{2} s^{2}\right)}-\frac{\beta}{\pi} \int_{\gamma_{1}}^{\gamma_{2}} \frac{\phi(u)\left(1-u^{2}\right)^{1 / 2}}{u-s} \mathrm{~d} u\right\}, \quad-1<s<1 .
$$

Substituting $\psi(s)$ from (25) into (24), we obtain

$$
\begin{gathered}
\frac{1}{\pi} \int_{\gamma_{1}}^{\gamma_{2}} \frac{\phi(u)}{u-s} \mathrm{~d} u-\frac{\beta^{2}}{\pi\left(1-s^{2}\right)^{1 / 2}} \int_{\gamma_{1}}^{\gamma_{2}} \frac{\phi(u)\left(1-u^{2}\right)^{1 / 2}}{u-s} \mathrm{~d} u=\frac{T}{C\left(1+d^{2} s^{2}\right)}\left[1-\frac{\beta S s\left(1+d^{2}\right)^{1 / 2}}{T\left(1-s^{2}\right)^{1 / 2}}\right] \\
\gamma_{1}<s<\gamma_{2} .
\end{gathered}
$$

The problem is now reduced to the solution of the Cauchy type singular integral equation (26) with the constraint (22). Equation (26) can be easily put in the form of an integral equation with a strictly Cauchy and a regular kernel. The case of a single interface crack is obtained in the limit $h \rightarrow \infty$ or $d \rightarrow 0$ and confirmed by the respective equation in [6]. It is noted that the function $\phi(u)$ is bounded and vanishes at the end points of $\gamma_{1}$ and $\gamma_{2}$.

The stress intensity factors for shear $K_{2}( \pm L)$ can be computed from $B_{x}(x)$ as [4]

$$
K_{2}( \pm L)= \pm C \lim _{x \rightarrow \pm L}\left[\left(L^{2}-x^{2}\right)^{1 / 2} B_{x}(x)\right]
$$

or in the new variables using (19) and (25)

$$
\frac{K_{2}( \pm L)}{C L^{1 / 2}\left[\frac{2 h d}{\pi L\left(1+d^{2}\right)}\right]^{1 / 2}}=\frac{S}{C\left(1+d^{2}\right)^{1 / 2}} \mp \frac{\beta}{\pi} \int_{\gamma_{1}}^{\gamma_{2}} \frac{\phi(u)\left(1-u^{2}\right)^{1 / 2}}{u \mp 1} \mathrm{~d} u .
$$

The normal stress intensity factors $K_{1}$ are given in terms of $K_{2}[4]$

$$
K_{1}\left( \pm L^{-}\right)=\mp \beta K_{2}( \pm L) \text {. }
$$

It is also recalled from [4] that $K_{1}$ is zero ahead of the crack tips $\pm L$.

\section{NUMERICAL RESULTS}

The numerical procedure employed for the solution of the singular integral equation (26) with the constraint (22) is explained in detail in [5] and [6], and only the results are discussed here. It suffices to mention that the direct integration method developed by Erdogan and Gupta [9] was applied. As in [6], it was found that for positive $\beta$ the left contact zone is very small, or of the order of $10^{-7} \mathrm{~L}$. Left contact zone values ranging from $10^{-6} \mathrm{~L}$ to $10^{-8} \mathrm{~L}$ do not appreciably affect the solution. Thus, in all the results presented here, the left contact zone was kept fixed and equal to $(0.8) 10^{-6} \mathrm{~L}$. The results were generated for $\beta=0.5$ and various values of the load and periodicity parameters $T / S$ and $h / L$. Negative $T / S$ indicates compression.

Figures 2 and 3 show the dimensionless shear stress intensity factors $K_{2}( \pm L) / S L^{1 / 2}$ as functions of $T / S$ for various values of $h / L$. The curve for $h / L=\infty$ corresponds to the single interface crack [6]. It is seen that the stress intensity factors decrease as the spacing between the cracks decreases, indicating that the cracks do not have a tendency to join and form larger cracks. The normal tractions $\sigma_{y y} / S$ in the larger contact zone are shown in Figs. 4 and 5 for $T / S=0.4$ and -0.4 , respectively. The curves exhibit the singular behavior of the contact stress as $x \rightarrow L^{-}$. The gaps or crack opening displacements afe plotted for various values of $h / L$ in Figs. 6 and 7 for $T / S=0.4$ and -0.4 , respectively. These figures show that as $h / L$ increases, the right contact zone decreases, while the contact pressure is locally relieved at $b$. 
$\sum^{\infty}$
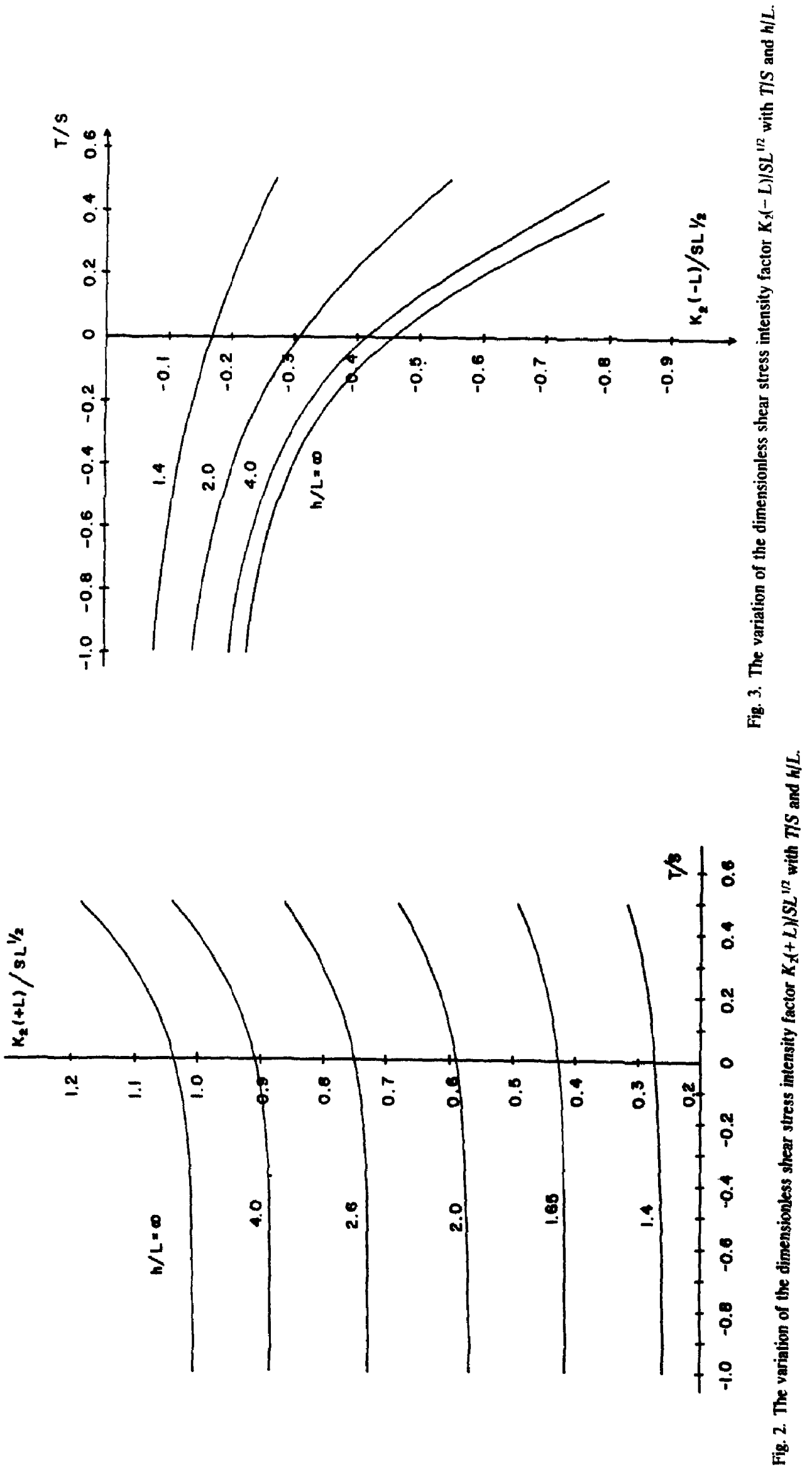


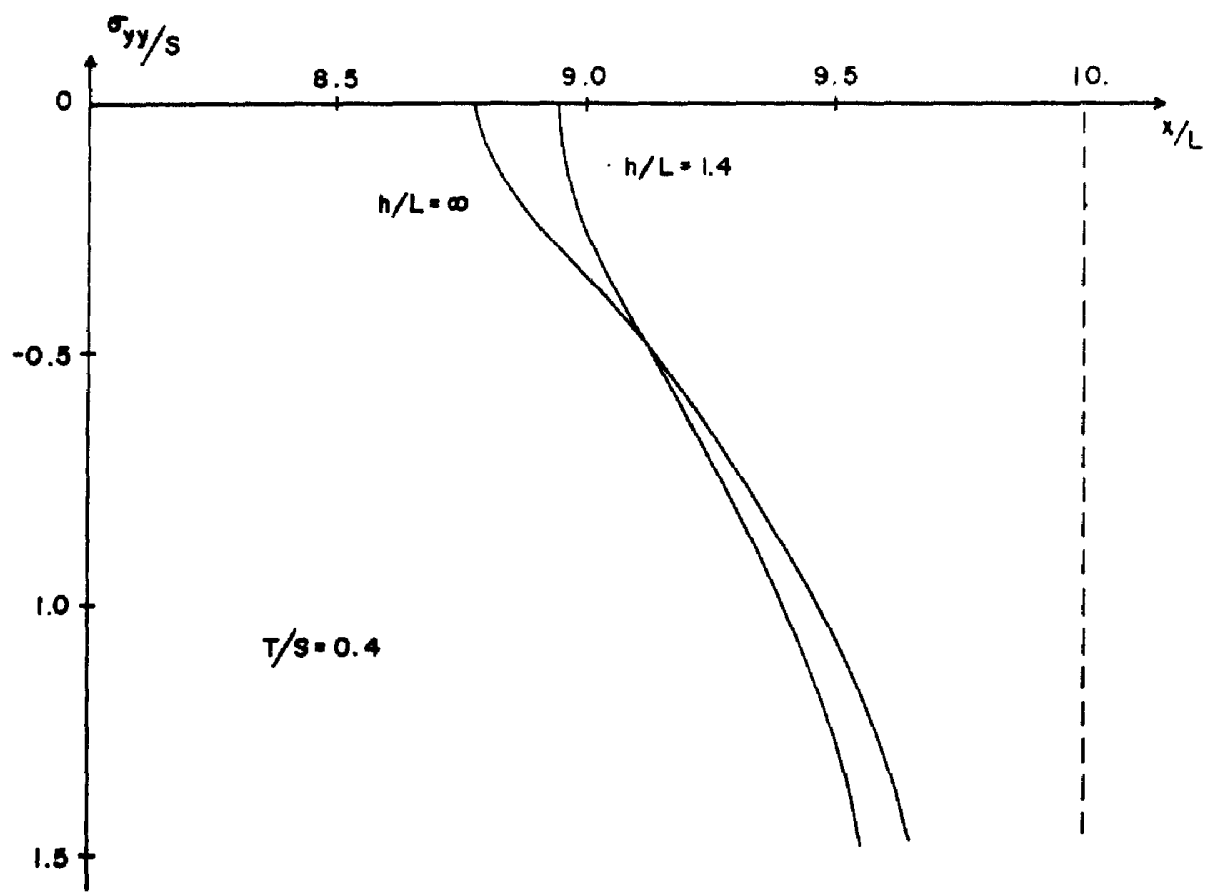

Fig. 4. Normal traction $\sigma_{y y} / S$ in the right contact zone for $T / S=0.4$.

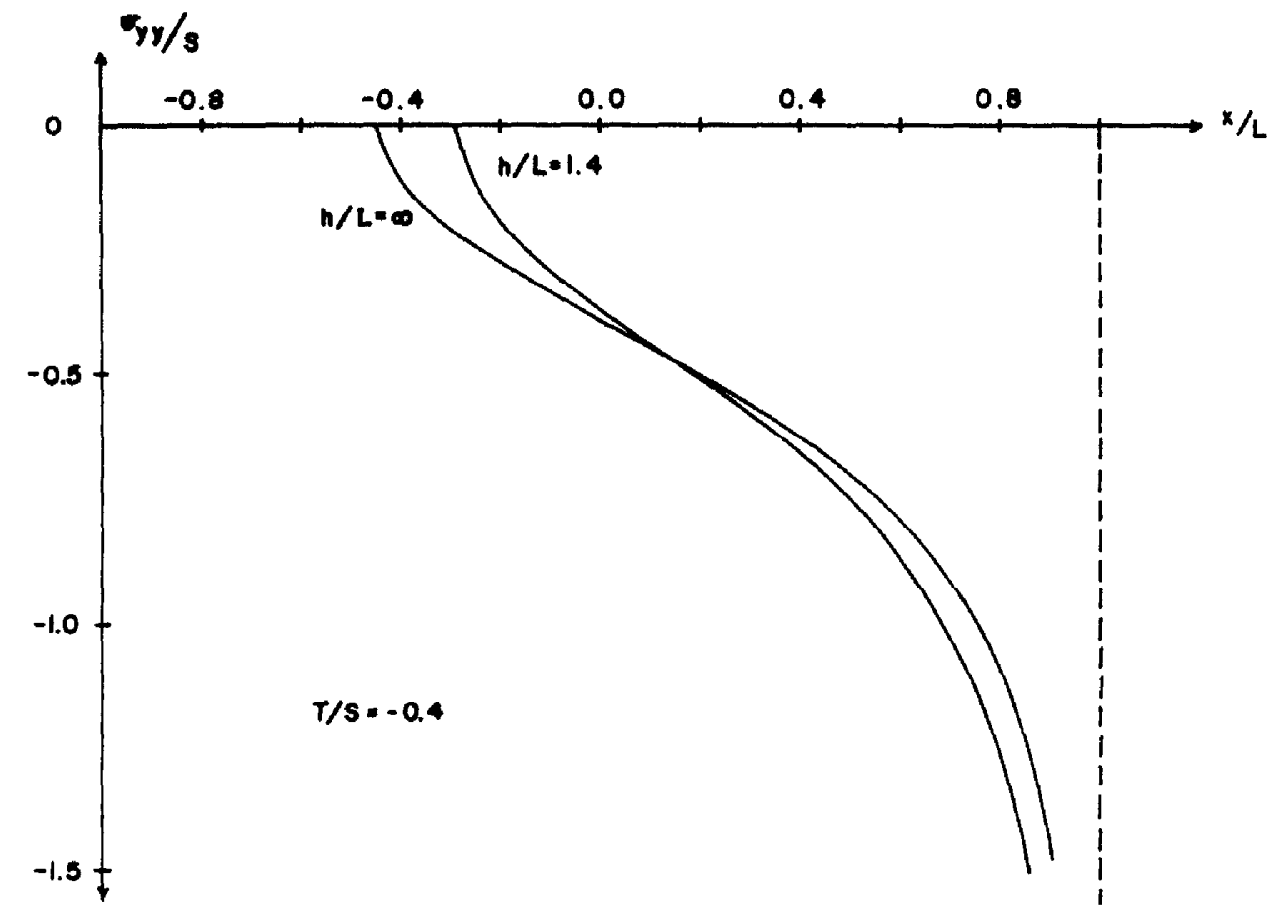

Fig. 5. Normal traction $\sigma_{v v} / S$ in the right contact zone for $T / S=-0.4$. 


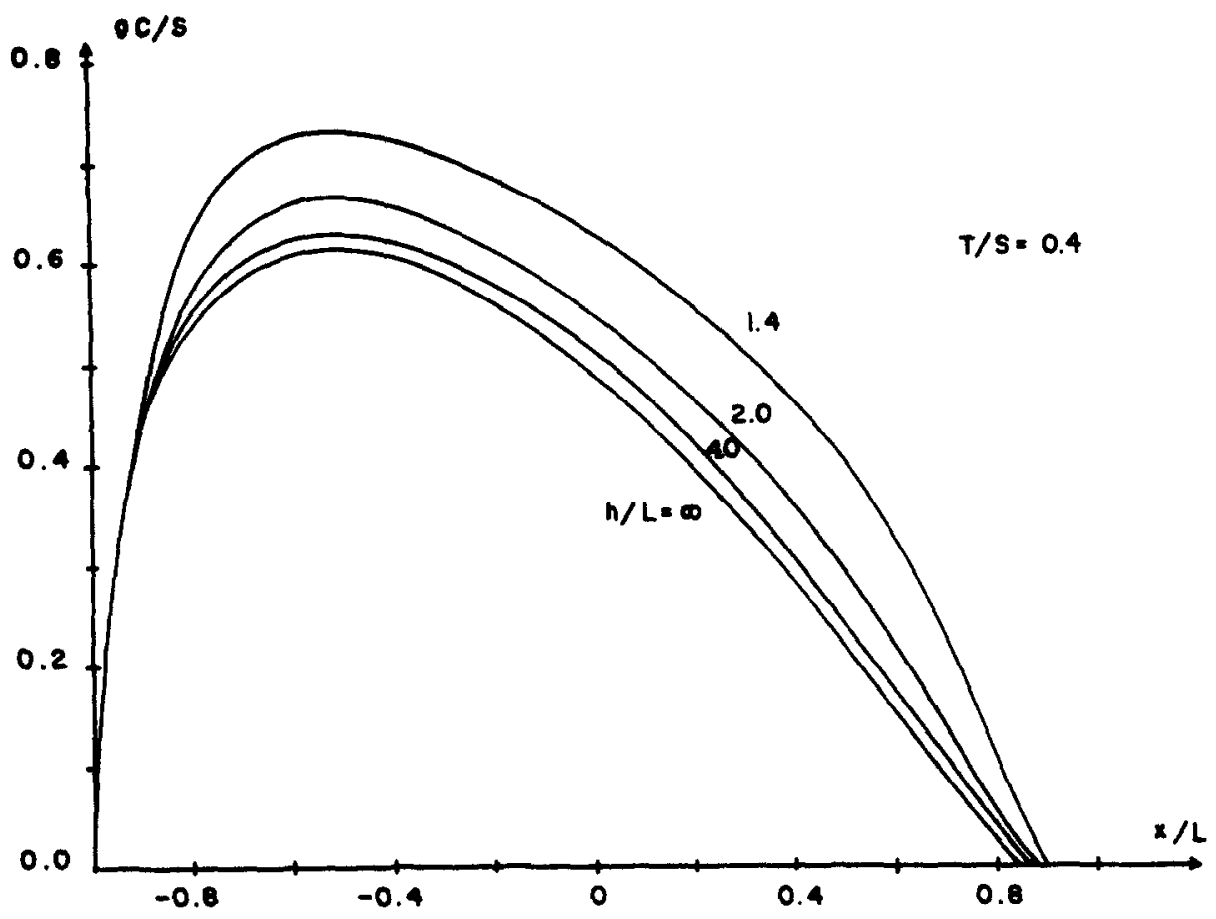

Fig. 6. Crack opening displacement $g C / S$ for $T / S=0.4$ and various values of $h / L$.

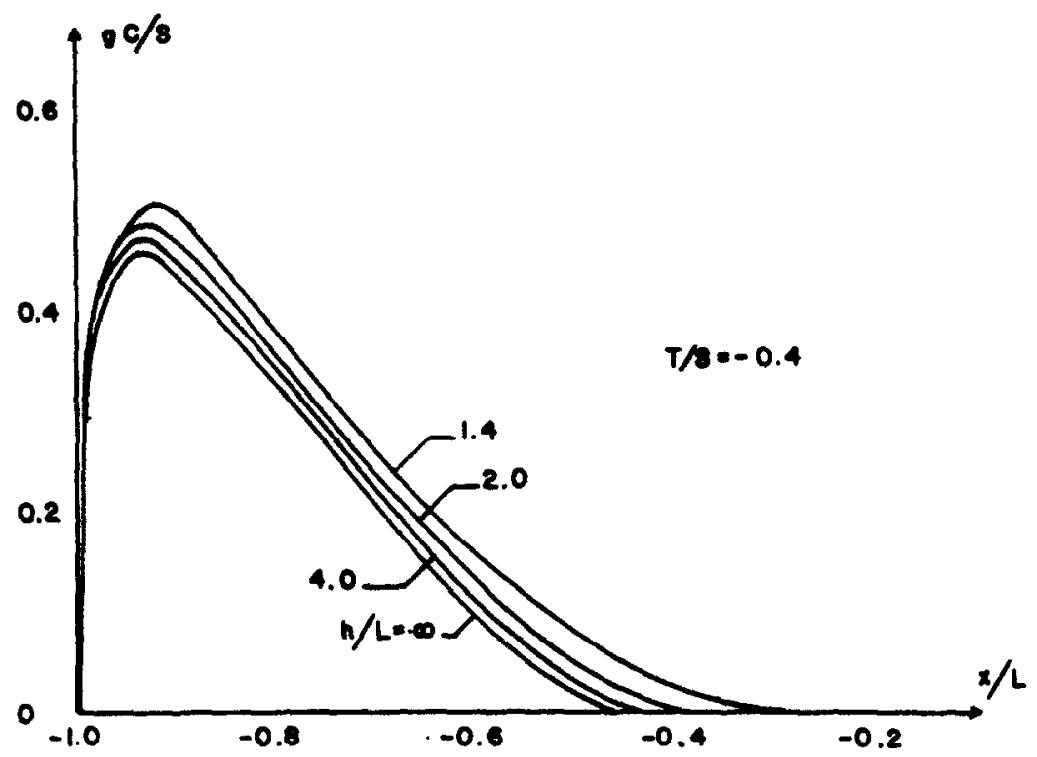

Fig. 7. Crack opening displacements \&CIS for $T / S=-0.4$ and various values of $h / L$.

Acknowledgement-One of the authors (M.C.) ernefully acknowlediges the expport by the National Science Foundation throust the grant ENG-772s032 during the course of this research.

\section{REFERENCES}

1. F. Erdogen, Stress diatribution in a nonhowneneous elastic phane with cracks. J. Appl. Mech, 31, 232 (1963).

2. F. Erdogan, Stress distribution in bondod dissimilar materisls with cracks. J. Appl. Mach. 32 is (1965).

3. J. R. Willis, The penny-ehaped crack on an interface. Quart. J. of Mech. Appl. Meth. 25, 367 (1972).

4. M. Comainou, The interface crack. J. Appl. Mech. 44,631 (1977).

5. M. Comninou, The interface crack in a shear field. J. Appl. Mech. 45, 287 (1978). 
6. M. Comninou and D. Schmueser, The interface crack in a combined tension-compression and shear field. J. Appl. Mech. 46. 345 (1979).

7. I. S. Gradshteyn and I. M. Ryzhik, Tables of Integrals, Series and Products. Academic Press, New York (1965).

8. N. I. Muskelishvili, Singular Integral Equations. Noordhoff, Leyden (1953).

9. F. Erdogan and G. Gupta, On the numerical solution of singular integral equations. Quart. Appl. Math. 29, 525 (1972). 